\title{
Hard X-ray emission from the Galactic ridge ${ }^{\star}$
}

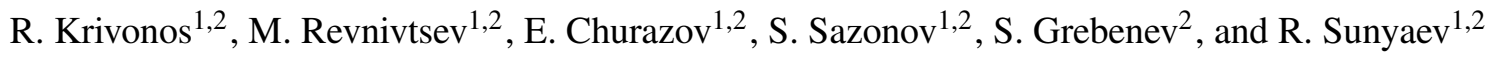 \\ 1 Max-Planck-Institute für Astrophysik, Karl-Schwarzschild-Str. 1, 85740 Garching bei München, Germany \\ e-mail: krivonos@mpa-garching.mpg.de \\ 2 Space Research Institute, Russian Academy of Sciences, Profsoyuznaya 84/32, 117997 Moscow, Russia
}

Received 17 May 2006 / Accepted 13 October 2006

\begin{abstract}
We present results of a study of the Galactic ridge X-ray emission (GRXE) in hard X-rays performed with the IBIS telescope aboard INTEGRAL. The imaging capabilities of this coding aperture telescope make it possible to account for the flux from bright Galactic point sources whereas the wide field of view permits us to collect large flux from the underlying GRXE. Extensive study of the IBIS/ISGRI detector background allowed us to construct a model that predicts the detector count rate with $\sim 1-2 \%$ accuracy in the energy band 17-60 keV. The derived longitude and latitude profiles of the ridge emission are in good agreement with the Galactic distribution of stars obtained from infrared observations. This, along with the measured hard X-ray spectrum of the Galactic ridge emission strongly indicates its stellar origin. The derived unit stellar mass emissivity of the ridge in the energy band 17-60 keV, $(0.9-1.2) \times 10^{27} \mathrm{erg} \mathrm{s}^{-1} M_{\odot}^{-1}$ (assuming a bulge mass of $1-1.3 \times 10^{10} M_{\odot}$ ), agrees with that of local (in the Solar neigborhood) accreting magnetic white dwarf binaries - dominant contributors to the GRXE at these energies. In addition, the shape of the obtained GRXE spectrum can be used to determine the average mass of white dwarfs in such systems in the Galaxy as $\sim 0.5 M_{\odot}$. The total hard $\mathrm{X}$-ray luminosity of the GRXE is $L_{17-60 \mathrm{keV}}=(3.7 \pm 0.2) \times 10^{37} \mathrm{erg} \mathrm{s}^{-1}$ in the $17-60 \mathrm{keV}$ band. At energies $70-200 \mathrm{keV}$ no additional contribution to the total emission of the Galaxy apart from the detected point sources is seen.
\end{abstract}

Key words. Galaxy: structure - Galaxy: bulge - Galaxy: disk - X-rays: diffuse background - stars: white dwarfs - X-rays: general

\section{Introduction}

Broad-band studies of the radiative output of the Galaxy demonstrate that different physical mechanisms contribute to the brightness of the Galaxy in different energy bands. In the nearinfrared and optical spectral bands, the bulk of the emission is provided by different types of stars. In the high energy ( $h v>$ $\mathrm{GeV}$ ) band the Galactic emission is likely a result of interactions of cosmic rays with interstellar matter (e.g., Kraushaar et al. 1972; Stecker 1973, 1977; Kniffen et al. 1978; Hunter et al. 1997).

From the first all sky surveys in X-rays $(\sim 2-10 \mathrm{keV})$ it became clear that in this energy band the emission of the Galaxy as a whole is dominated by the contribution from bright point sources, mainly accreting black holes and neutron star binaries. However, emission that was not resolved into separate point sources - the Galactic ridge X-ray emission (GRXE, e.g. Worrall et al. 1982) - was also discovered. Even the significant increase of the sensitivity of X-ray instruments over the last decades has not led to resolving all the Galactic ridge emission into discrete sources (Sugizaki et al. 2001; Hands et al. 2004; Ebisawa et al. 2005). This was considered as an indication of a truly diffuse origin of the Galactic ridge emission.

The latest studies of the morphology and volume emissivity of the GRXE in the energy band 3-20 keV provide convincing evidence that the majority of the GRXE consists of a large number of stellar type X-ray sources, namely white dwarf binaries

* Based on observations with INTEGRAL, an ESA project with an instrument and science data center funded by ESA member states (especially the PI countries: Denmark, France, Germany, Italy, Switzerland, Spain), The Czech Republic, and Poland, and with the participation of Russia and the USA. and coronally active stars (Revnivtsev et al. 2005; Sazonov et al. 2006). In particular for the energy band $>20 \mathrm{keV}$, this means that the GRXE must be dominated by the contribution of magnetic white dwarf binaries - intermediate polars (IP) and polars (P). Assuming that the GRXE traces the stellar mass density in the Galaxy, one can obtain a proxy of the GRXE spectrum in hard X-rays (20-200 keV) from the spectrum of the inner $30 \mathrm{pc}$ $\left(\sim 12^{\prime}\right)$ of our Galaxy (Revnivtsev et al. 2005). However, since the Galactic center region may be peculiar in many respects, study of the true GRXE in hard X-rays is necessary.

The GRXE spectrum above $>20 \mathrm{keV}$ is not yet accurately measured (e.g., Purcell et al. 1996; Skibo et al. 1997; Kinzer et al. 1999). The most recent results on GRXE were obtained with the instruments aboard INTEGRAL observatory (Winkler et al. 2003). Lebrun et al. (2004) and Terrier et al. (2004) using IBIS telescope (Ubertini et al. 2003) have shown that although bright point sources dominate the emission from the Galaxy at $30 \mathrm{keV}$, there is a significant unresolved component. Bouchet et al. (2005) and Strong et al. (2005) using SPI spectrometer (Vedrenne et al. 2003) found an unresolved Galactic X-ray emission at energies higher than $50 \mathrm{keV}$.

To determine the origin of the hard X-ray Galactic background it is very important to investigate whether the GRXE in hard X-rays is distributed similar to the stellar distribution, indicating its stellar origin, or whether it more closely follows the interstellar gas density distribution, thus connecting to the high energy gamma-ray background seen, e.g., by EGRET. Either the spectrum of the GRXE has a cutoff at energies $\sim 30-50 \mathrm{keV}$ due to the typical cutoff in spectra of magnetic CVs (e.g., Suleimanov et al. 2005), or it has a power-law spectral shape up to higher energies as would be expected if the Galactic background emission were induced by cosmic ray electrons 
(e.g., Stecker 1977; Mandrou et al. 1980; Sacher \& Schoenfelder 1984; Skibo \& Ramaty 1993).

Previous attempts to study the hard X-ray component of the GRXE were severely inhibited by the poor angular resolution of the instruments used, which precluded effective subtraction of the contribution of bright point sources. Only now has this become possible thanks to the hard X-ray telescopes aboard the INTEGRAL observatory. The IBIS telescope on INTEGRAL possesses an optimal combination of properties to perform such a study:

- it has a relatively large field of view $\left(\sim 28^{\circ} \times 28^{\circ}\right.$ at zero response) that allows a large flux of the diffuse emission to be collected, but not too large to preclude the construction of a GRXE map if the telescope is used as a collimated instrument;

- it has the possibility of detecting and subtracting the contribution of point sources;

- its sensitivity to point sources for typical exposure times in the Galactic plane regions $\sim 1 \mathrm{Ms}$ is $\sim 10^{-11} \mathrm{erg} \mathrm{s}^{-1} \mathrm{~cm}^{-2}$, which for the Galactic center distance corresponds to a luminosity $\sim 10^{35} \mathrm{erg} \mathrm{s}^{-1}$. Subtraction of sources with luminosities higher than this limit allows one to avoid significant contamination of the GRXE by point sources (see, e.g., Sazonov et al. 2006).

Since its launch in 2002, INTEGRAL/IBIS has collected a large amount of observational data on different sky regions, and in particular on the inner Galactic plane where most of the GRXE is located.

In this work we will study the spectral and morphological properties of the GRXE in the hard X-ray energy band 17-200 keV. At higher energies the positronium annihilation continuum of the Galactic center (e.g., Leventhal et al. 1978; Gehrels et al. 1991; Churazov et al. 2005; Knödlseder et al. 2005) should be carefully taken into account, which requires a different approach from the one used in this work (which is especially related to the detector background modeling). For this reason we leave the study of the Galactic background emission at energies higher then $200 \mathrm{keV}$ for a separate paper.

\section{Data set and data filtering}

For our analysis we used all the IBIS data available to us, including public data, some proprietary data (Galactic center observations and Crux Spiral arm deep exposure observations) and data available to us through the INTEGRAL Science Working Team. In total we analyzed $\sim 33 \mathrm{Ms}$ of the data (deadtime corrected value of exposure). We considered only the data of the ISGRI detector of the IBIS telescope, which provides data in the energy band $\sim 17-1000 \mathrm{keV}$ with high sensitivity in hard X-rays $(17-200 \mathrm{keV})$ and has sufficient angular resolution $\left(\sim 12^{\prime}\right)$ for studying crowded fields like the Galactic center region.

The method of the sky reconstruction employed in the IBIS telescope (coded mask imaging) does not allow one to study directly diffuse structures that are significantly larger than the size of the mask pixels. Therefore, to study large-scale structures, such as the GRXE $\left(\sim 100^{\circ} \times 5^{\circ}\right)$, we should use IBIS/ISGRI as a collimated instrument. The detector collects photons from point sources and diffuse emission. Measurement of the point source' contribution to the total detector count rate makes it possible to recover the flux of the GRXE. The success of such approach strongly depends on the accuracy of the instrumental background modeling.
Prior to subsequent analysis we screened the data. If an individual observation (SCW - "science window") did not fulfill all the imposed criteria it was dropped. However, we should note that screening of individual events is likely more flexible and could have saved slightly more data. We screened all the data near the beginning and end of revolutions (due to increased background near the radiation belts), the data when ISGRI was operated not in its main regime (modes 41 and 43), and science windows with exposure times less then $\sim 700 \mathrm{~s}$. We also applied filtering using information about the electron count rate provided by IREM (INTEGRAL Radiation Environment Monitor, Hajdas et al. 2003). Analysis of the detector 10-s binned light curve in the energy band $17-200 \mathrm{keV}$ was used to screen science windows with all types of bursts. As a final step in the screening procedure, we filtered out all observations that had a high level of noise on source-free images (sky images with removed point sources). Upon the data filtering only $\sim 60 \%$ of observations were accepted for further analysis.

\section{Detector modeling}

\subsection{Detector background}

At any given time the detector count rate of IBIS/ISGRI consists of:

- Cosmic X-ray Background (CXB);

- emission from point sources;

- galactic ridge X-ray emission, if the field of view of the telescope is directed towards the Galactic plane;

- detector internal background, caused by different processes including activation of different elements of the spacecraft, interaction of the detector material with cosmic-rays, etc. (e.g., Bloser et al. 2002; Terrier et al. 2003).

The cosmic X-ray background contributes $\sim 0.5 \mathrm{Crab}$ in the energy band 17-100 keV and can be considered very uniform over the sky. Taking into account the IBIS/ISGRI field of view, we can expect that CXB flux variability over the sky for ISGRI will not exceed $\sim 1 \%$ (e.g., Churazov et al. 2006). Therefore, the contribution of the CXB to the detector count rate can be considered independent of observation orientation and can be estimated from observations at high Galactic latitudes. The contribution of point sources to the detector count rate can be almost perfectly predicted using the telescope coded mask imaging technique (more on this below). The list of detected sources used in our subtraction procedure includes more than 360 sources on the entire sky. Typically, the detection limit for regions near the Galactic plane is at the level of $\sim 1 \mathrm{mCrab}$. The complete list of sources will be presented elsewhere. To predict the detector count rate not caused by photons arriving from the sky one should use a background model. We followed two different approaches to study the ridge morphology and its energy spectrum. For the study of the energy spectrum we used only specially performed INTEGRAL observations for which the systematical uncertainties are minimal (see description below, model 2), whereas for the study of the ridge emission distribution in the Galaxy we used all the available observations.

\section{Model 1: Background model using tracers}

Among possible tracer candidates that might be used in the background modeling, we considered the IREM count rates, SPI saturated events count rates, ISGRI veto count rates, and ISGRI high energy band count rates. We finally chose the ISGRI detector count rate in the energy band 600-1000 keV. At these 


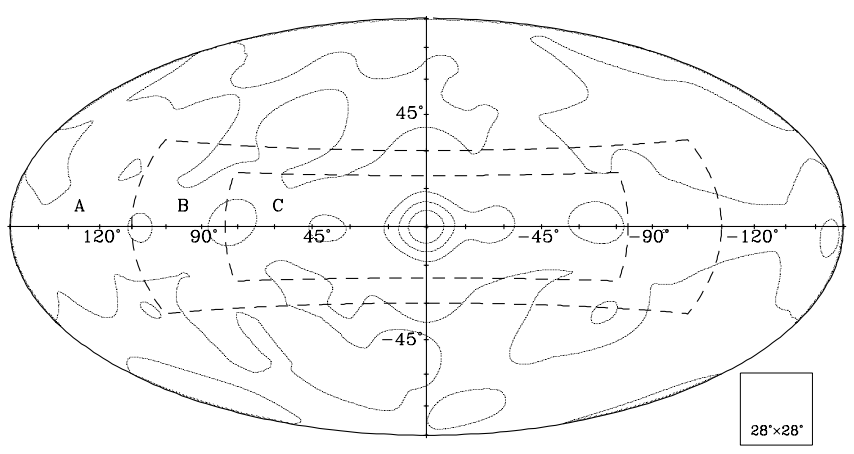

Fig. 1. Labeling of the sky areas used in this work. Area "A" $\left(|l|>120^{\circ}\right.$ and $|b|>30^{\circ}$, excluding a $30^{\circ}$-radius circle around the Crab nebula) and "B" $\left(90^{\circ}<|l|<120^{\circ}\right.$ and $\left.20^{\circ}<|b|<30^{\circ}\right)$ contain extragalactic fields used for calibration of the background model. Area "B" was also used to estimate the accuracy of the method. The Galactic ridge $\mathrm{X}$-ray emission was studied in field "C" observations $\left(|l|<80^{\circ}\right.$ and $|b|<20^{\circ}$ ). Contours of the total (deadtime corrected) exposure time are shown overimposed. The contour levels correspond to 5.3, 3.5, and $1.8 \mathrm{Ms}$, and $50 \mathrm{ks}$. The IBIS FOV is shown for comparision at the right bottom corner of the plot.

energies the effective area of the ISGRI detector is very small $\left(<40 \mathrm{~cm}^{2}\right)$, and the detector count rate is expected to be dominated by the internal detector background. We also took into account gain variations of the ISGRI detector, which shift the detector background spectrum along the energy axis causing additional variations of the count rates in the studied energy bands. As we are mainly interested in the energies $17-200 \mathrm{keV}$, we estimated the ISGRI detector gain by observing the position of strong background lines at energies $\sim 60 \mathrm{keV}$ (e.g., Terrier et al. 2004).

Our final model of the ISGRI background consists of a linear combination of the 600-1000 keV detector count rate $H$ and the gain parameter $G$. To make allowance for possible long-term variations of the ISGRI detector background, we also included time $(T)$ in the cubic polynomial form:

$S=a_{0}+a_{1} H+a_{2} G+a_{3} T+a_{4} T^{2}+a_{5} T^{3}$.

The coefficients in Eq. (1) for the 17-60 keV energy band were calculated using observations pointed away from the Galactic center and away from the inner Galactic plane, where the GRXE is negligible (Revnivtsev et al. 2005) - regions " $A$ " and " $B$ " in Fig. 1 (hereafter we consider only the detector count rates after removal of the contribution of point sources).

To test the background model we derived coefficients for our model using pointings in region "A" Fig. 1 and applied the model to pointings from region "B". The result of the background model implementation is presented in Fig. 2 (bottom panel). The employed background model leaves residuals with an rms scatter $\sim 1.8 \%$ of the mean background level (see bottom panel in Fig. 2). However, it should be noted that this rms scatter contains significant contribution from the pure statistical variation of the (point sources removed) detector count rate. To reduce the contribution of this statistical scatter and to reveal only systematic uncertainties we averaged the measured field "B" residuals (differences between the measured detector count rates and model predictions) over the Galactic latitude. The resulting residuals are presented in Fig. 3. The root-mean-squared value of the residuals is $1.1 \times 10^{-4} \mathrm{cnts} / \mathrm{sec} / \mathrm{pix}$ in the energy band $17-60 \mathrm{keV}$ ( $\sim 1 \%$ of the detector background), which approximately corresponds to a flux $\sim 10 \mathrm{mCrab}$ for a Crab-like spectrum.

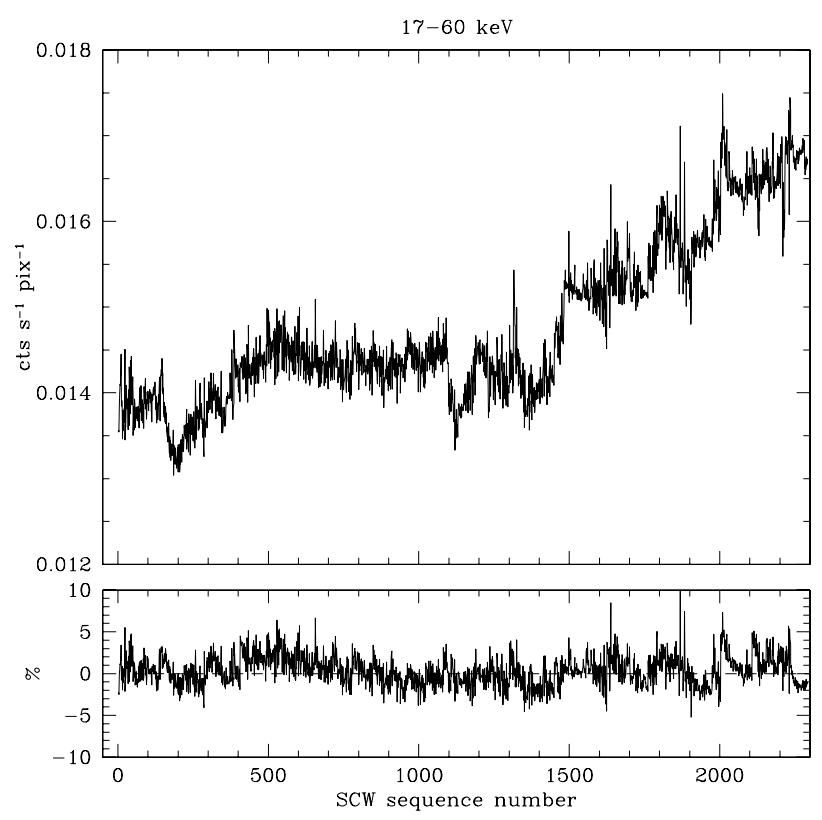

Fig. 2. Top panel: ISGRI count rate in the $17-60 \mathrm{keV}$ energy band as a function of SCW sequence number. The pointings were selected in area "B" (see Fig. 1). Bottom panel: residuals after subtracting the model-predicted count rate from the observed count rate (in percent with respect to the observed count rate).

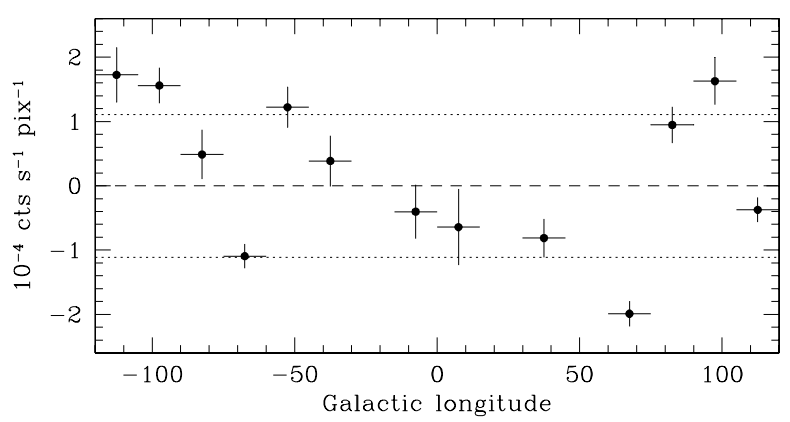

Fig. 3. Residuals of the detector count rate of field "B" observations from the background model averaged over Galactic longitude. Dotted lines represent a $1 \sigma$ deviation $\left(1.1 \times 10^{-4} \mathrm{cts} \mathrm{s}^{-1}\right.$ pix $\left.^{-1}\right)$ of averaged values from zero.

\section{Model 2: Rocking mode}

Part of the Galactic center region observations - Galactic center latitude scans (March 2005-March 2006) - were taken by INTEGRAL using a specially designed pattern, which presents considerable advantages from the point of view of the study of the Galactic ridge energy spectrum. The pointing direction of INTEGRAL instruments were moved across the Galactic center region on a timescale $\sim 10 \mathrm{~h}$, which is smaller than that of significant changes of the INTEGRAL/IBIS/ISGRI instrumental background. This mode of observations turns the IBIS/ISGRI instrument into some kind of a rocking collimator experiment. Therefore, the prediction of the ISGRI/IBIS instrumental background in this mode of observations was calculated using interpolation between ISGRI flux measurements done at high $\left(|l|>20^{\circ}\right)$ Galactic latitudes, where the surface brightness of the Galactic background emission is negligible (e.g., Revnivtsev et al. 2005)

We checked the quality of the ISGRI instrumental background subtraction on high energy channels $(>600 \mathrm{keV})$ where the instrumental background totally dominates. We found that 

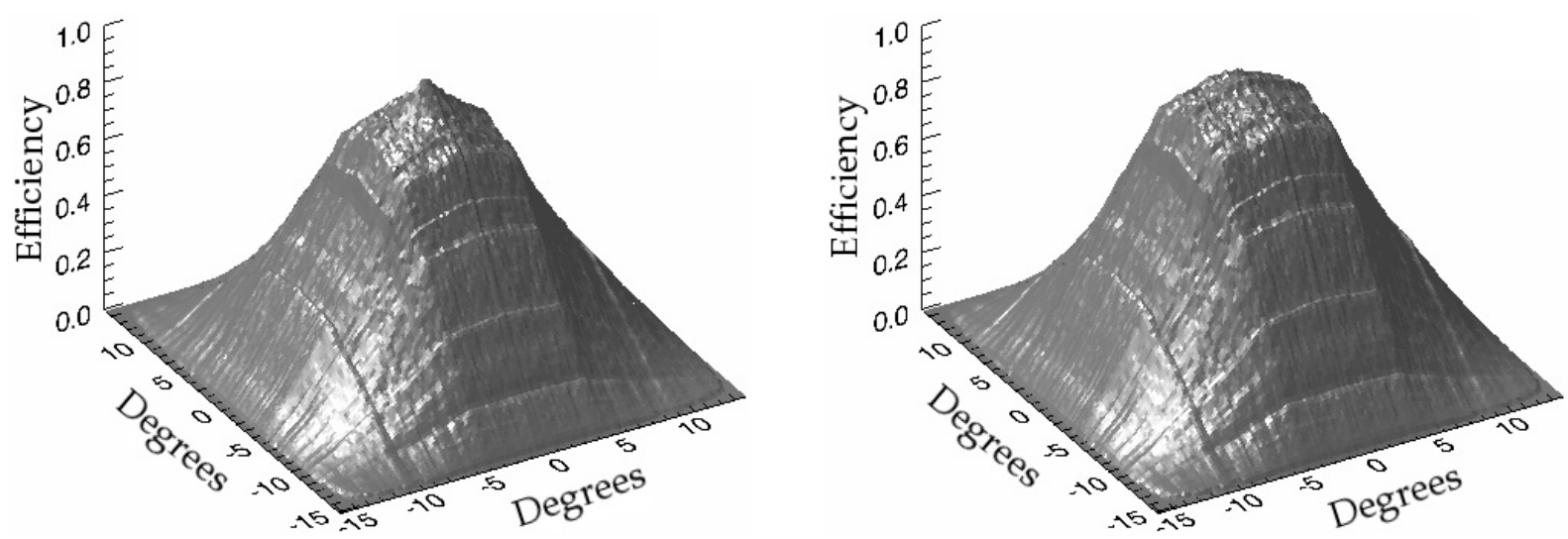

Fig. 4. IBIS/ISGRI efficiency as a function of point source position within the field of view. The model of the IBIS/ISGRI efficiency in the energy band 17-60 keV (left panel) and 86-129 keV (right panel). Observations of the Crab nebula were used for the calibration.

the systematical uncertainties of the background subtraction using the employed technique $(\sim 0.5 \%$ of the detector count rate) do not exceed the statistical uncertainties of the $\sim 1 \mathrm{Ms}$ dataset used. In particular, for energy channels $\sim 100-200 \mathrm{keV}$ this means approximately 7 times better quality of the background subtraction than when using the method described in previous paragraphs (model 1) $-\sim 15$ and $\sim 100$ mCrab correspondingly. Thus for the construction of the Galactic ridge energy spectrum we used only these observations. Unfortunately this method cannot be used for studying the whole Galaxy because the special pattern of observations (Galactic latitude scans) is available only for the Galactic center region.

\subsection{Accuracy of the sources subtraction, IBIS collimator efficiency, and influence of the IBIS mask transparency}

One of the key steps in studying the GRXE with the large field of view instrument IBIS/ISGRI is the subtraction of the contribution of bright point sources from the detector count rate. The unsubtracted count rate due to bright point sources can significantly distort the map of the GRXE and its energy spectrum. There are two main constituents of this problem: imperfect mask shadow modeling in the software, which will result in leftover unsubtracted count rate on the detector, and the finite opacity of the IBIS mask, which will lead to an underestimation of source count rates by the coded mask technique and therefore to an unsubtracted source count rate on the detector. To check the quality of our source removal procedure and the accuracy of our constructed IBIS collimator response function, we studied a number of observations of the Crab nebula. Our model of the IBIS/ISGRI collimator response function includes a geometry of the instrument and also an angle and energy dependent absorption caused by the "NOMEX" structure, supporting IBIS mask. (see, e.g., Reglero 2001). Examples of used response functions in the energy bands $17-60 \mathrm{keV}$ and $86-129 \mathrm{keV}$ are presented in Fig. 4.

The residuals between the predicted count rates during Crab observations (which include the results of our background model and our collimator response function model) and the actually measured detector count rates do not demonstrate any dependence on source flux and the rms scatter of the residuals does not exceed the uncertainty of the our detector background model estimated in Sect. 3.1. Therefore, we can conclude that neither our source removal procedure nor finite mask opacity in the energy range 17-200 keV introduced additional systematic uncertainties into our analysis.

\section{Results}

Using the method described above for each INTEGRAL/IBIS/ ISGRI observation we obtain two numbers in any considered energy channel: 1) the summed detector count rate caused by resolved point sources and 2) the detector count rate left after subtraction of the contribution of point sources and modeled detector background. The possible remaining contribution of undetected point sources on the detector can be estimated using the luminosity function of Galactic X-ray sources (Grimm et al. 2002; Sazonov et al. 2006). The majority of the inner Galactic plane was observed by INTEGRAL/IBIS for more than 0.5-0.8 Ms. Such an exposure corresponds to an IBIS/ISGRI detection sensitivity $\sim 1 \mathrm{mCrab} \sim 10^{-11} \mathrm{erg} \mathrm{s}^{-1} \mathrm{~cm}^{-2}$ in the energy band 17-60 keV, which in turn corresponds to a source luminosity $\sim 10^{35} \mathrm{erg} \mathrm{s}^{-1}$ for the Galactic center distance. The contribution of sources brighter than this limit was subtracted from the detector count rate. Therefore, from Fig. 12 of Sazonov et al. (2006) we can conclude that the contribution of undetected point sources does not significantly affect the emission of the GRXE.

\subsection{Morphology}

We constructed longitude and latitude profiles of the integrated emission from point sources and of the hard X-ray Galactic ridge emission. For construction of the longitude profile of the GRXE we selected those INTEGRAL observations where the IBIS axis was directed within $5^{\circ}$ of the Galactic plane and then averaged the obtained GRXE flux measurements in nearby longitude bins. The obtained profile is presented in Fig. 5. The blue (upper) histogram represents the summed flux of point sources seen by the IBIS/ISGRI detector. Error bars on this histogram represent the rms deviations of the individual measurements of the summed point source fluxes. On this plot one can clearly see the contributions of a number of sources in the Galactic center region $\left(l \sim 0^{\circ}\right)$ and well-known bright Galactic X-ray sources Cyg X-1 $\left(l \sim 70^{\circ}\right)$, GRS $1915+105\left(l \sim 45^{\circ}\right)$, and GX 301-2 $\left(l \sim-60^{\circ}\right)$. The latitude profiles (detected sources and unresolved background) were obtained by averaging those GRXE flux measurements made when the IBIS telescope was directed within $|l|<5^{\circ}$ (Fig. 6). The contributions of bright Galactic center sources and Sco X-1 $\left(b \sim 20^{\circ}\right)$ are clearly seen.

As we used the IBIS/ISGRI with the field of view $\sim 15^{\circ} \times$ $15^{\circ}(F W H M)$ as a collimated instrument, the angular resolution of our resulting profiles is approximately $\sim 15^{\circ}$. This angular resolution is not ideal for studying the innermost (angular 


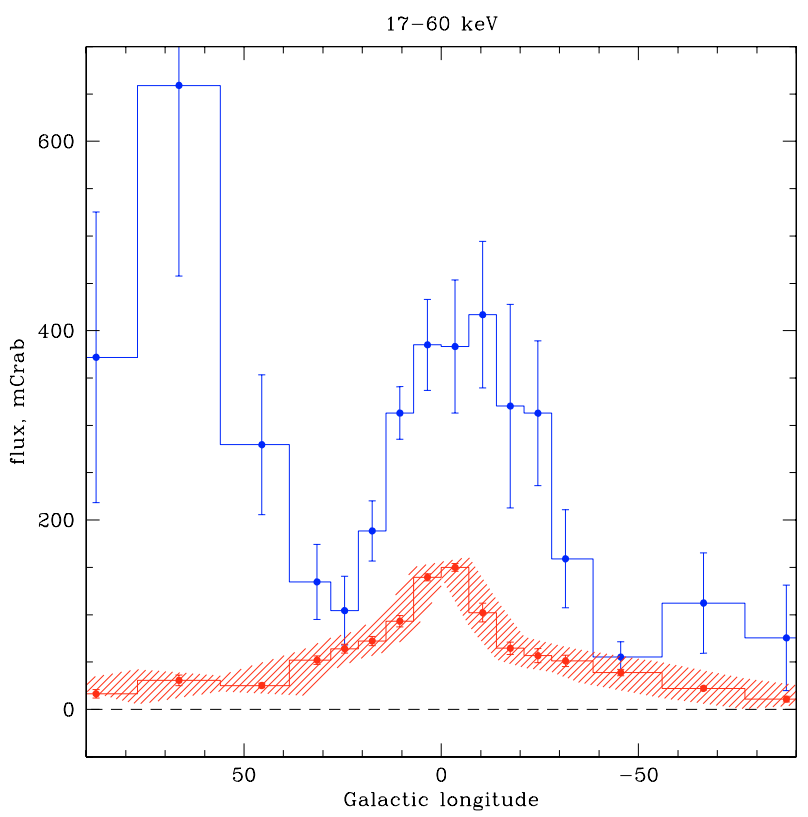

Fig. 5. Longitude profile of the ridge emission in the 17-60 keV energy range. Only observations during which the center of the ISGRI field of view was directed within $|b|<5^{\circ}$ were used for the construction of this profile. The profile is shown by the red histogram with the shaded region representing systematic uncertainties. The contribution of source emission is shown by the blue histogram. Error bars represent rms-deviations of individual measurements of the summed point source fluxes from the average flux values in bins.

scales $\left.1-2^{\circ}\right)$ regions of the Galaxy and latitude profiles of the GRXE (exponential scale heights $1.5-3^{\circ}$, see, e.g., Revnivtsev et al. 2005). Also taking into account the limited accuracy of our obtained IBIS collimator response function, we concluded that at the present level of uncertainties involved in our analysis we cannot make an accurate multiparameter model fitting of the GRXE volume emissivity. To extract information about the three-dimensional structure of the Galactic ridge in hard X-rays we have compared the profiles of GRXE proxies (with known properties) with those we obtained from INTEGRAL/ISGRI data. In particular, the current understanding of the GRXE morphology implies that the best tracer of the GRXE is the near infrared surface brightness (Revnivtsev et al. 2005).

The map of the Galaxy in the near infrared spectral band was obtained using data of COBE/DIRBE observations (zodi-subtracted mission average map provided by the LAMBDA archive of the Goddard Space Flight Center, http://lambda.gsfc.nasa.gov). To reduce the influence of the interstellar reddening we considered DIRBE spectral band $4.9 \mu \mathrm{m}$. We applied first-order corrections to the NIR map of the Galaxy obtained by COBE/DIRBE. We assumed that the intrinsic NIR color temperature (i.e., the ratio of intrinsic surface brightnesses $I_{1.2 \mu \mathrm{m}}$ and $I_{4.9 \mu \mathrm{m}}$ ) of the Galactic disk and the Galactic bulge/bar is uniform and its true value can be derived at high Galactic latitudes where the interstellar reddening is negligible. Then the foreground extinction map may be expressed as:

$A_{4.9 \mu \mathrm{m}}=\frac{-2.5}{A_{1.2 \mu \mathrm{m}} / A_{4.9 \mu \mathrm{m}}-1}\left[\ln \left(\frac{I_{1.2 \mu \mathrm{m}}}{I_{4.9 \mu \mathrm{m}}}\right)-\ln \left(\frac{I_{1.2 \mu \mathrm{m}}^{0}}{I_{4.9 \mu \mathrm{m}}^{0}}\right)\right]$.

Here the $A$ values are the reddening coefficients at different wavelengths. We have used the interstellar reddening values from works of Lutz et al. (1996) and Indebetouw et al. (2005).

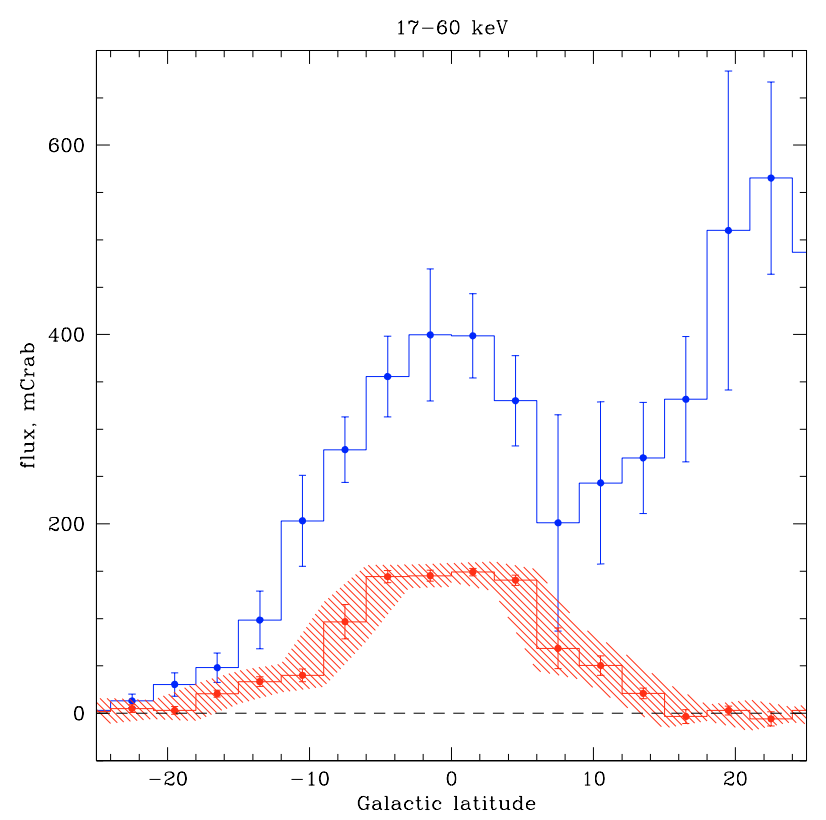

Fig. 6. Ridge emission latitude profile $\left(|l|<5^{\circ}\right)$. The plot description is the same as for Fig. 5.

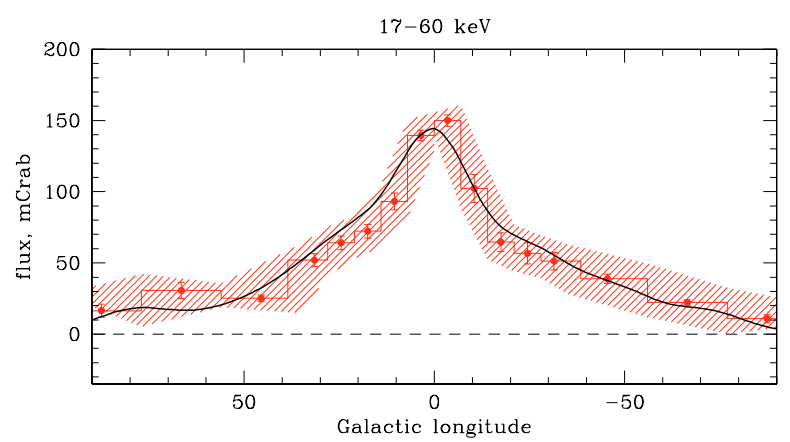

Fig. 7. Longitude profile of the GRXE measured by INTEGRAL/IBIS/ISGRI (histogram and shaded region) in the $17-60 \mathrm{keV}$ energy band along with the intensity profile of the Galactic NIR emission obtained by COBE/DIRBE at $4.9 \mu \mathrm{m}$ (solid line). The NIR map was convolved with the IBIS collimator response. Normalization of the NIR profile is determined from X-ray-NIR correlation function (see Fig. 10).

The employed correction of course removed only main effects of interstellar extinction on the COBE/DIRBE map, therefore we do not expect that the obtained COBE/DIRBE map and profiles have accuracy higher than $\sim 10 \%$.

The map of the NIR intensity was then convolved with the IBIS/ISGRI collimator response function (see Fig. 4). The resulting longitude and latitude profiles of the COBE/DIRBE NIR intensity are shown by the solid line on Figs. 7 and 8 correspondingly. We also constructed a map of the IBIS/ISGRI surface brightness distribution of the GRXE in the 17-60 keV energy band (see Fig. 9). The map of the NIR intensity is shown by contours. Correlation of observed values of hard X-ray flux with the NIR fluxes is presented in Fig. 10.

It is clearly seen that the GRXE intensity distribution very closely follows the NIR intensity distribution and thus traces the stellar mass density in the Galaxy. To show that the correlation of the hard X-ray GRXE with the cosmic-ray induced gammaray background emission is not nearly as good as its correlation with the NIR intensity, we present Fig. 11 . Here one can see the 


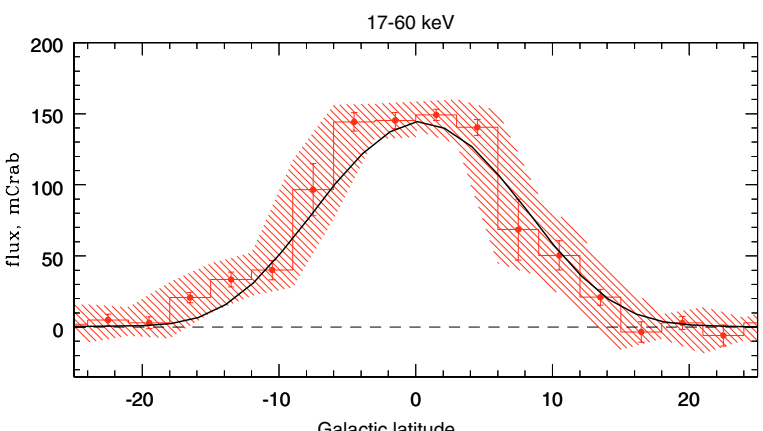

Fig. 8. Latitude profile of the GRXE. IBIS telescope pointings were selected within $|l|<5^{\circ}$. For plot description see Fig. 7.

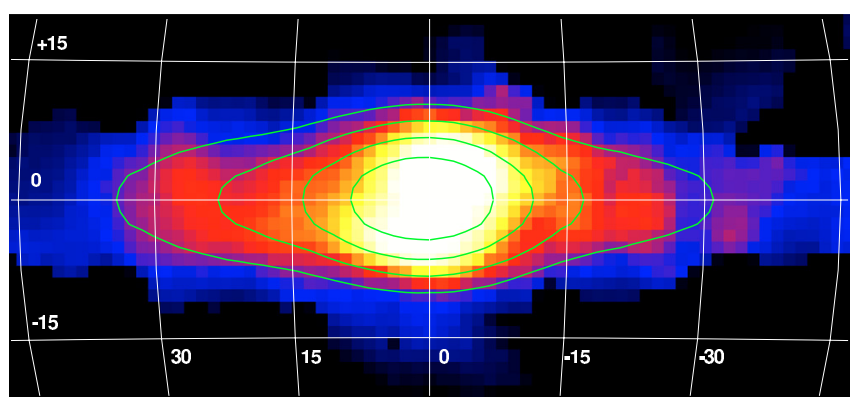

Fig. 9. Map of the Galactic diffuse emission observed by INTEGRAL/IBIS/ISGRI in the energy band 17-60 keV. Contours represent the near infrared intensity measured by COBE/DIRBE at $4.9 \mu \mathrm{m}$. NIR contours were convolved with the IBIS collimator response function. The contour levels correspond to 1.0,1.4, 1.8, $2.2 \times$ $10^{-5} \mathrm{erg} \mathrm{s}^{-1} \mathrm{~cm}^{-2}$ per IBIS FOV.

distributions (convolved with the IBIS/ISGRI collimator response function) of the EGRET gamma-ray background, Galactic neutral hydrogen (HI), and molecular gas (CO emission) $)^{1}$.

We can conclude that the emissivity profile of the GRXE in hard X-rays (17-60 keV) supports the finding of Revnivtsev et al. (2005) that the GRXE traces the stellar mass distribution. This allows us to estimate the emissivity of the GRXE in hard X-rays using the known NIR luminosity measured with COBE/DIRBE observations. The ratio of NIRand hard X-ray intensities averaged over the whole Galaxy is $F_{17-60 \mathrm{keV}} / F_{4.9 \mu \mathrm{m}}=(7.52 \pm 0.33) \times 10^{-5}$ (Fig. 10). The ratio derived using data of Galactic center latitude scans only (background model 2, see Sect. 3) is $F_{17-60 \mathrm{kev}} / F_{4.9 \mu \mathrm{m}}=(8.44 \pm$ $0.28) \times 10^{-5}$ (see Fig. 12) agrees with that measured over the whole Galaxy.

The ratios averaged over the Galactic bulge $\left(|l|<10^{\circ}\right)$ and the Galactic disk $\left(|l|>20^{\circ}\right)$ regions separately are $(7.73 \pm 0.34) \times$ $10^{-5}$ and $(6.53 \pm 0.72) \times 10^{-5}$ correspondingly (Fig. 13). Note that there is no statistically significant difference in the obtained ratios for the bulge and disk regions.

Using the $4.9 \mu \mathrm{m}$ luminosity of the Galactic bulge $L_{4.9 \mu \mathrm{m}}^{\text {bulge }}=$ $4.3 \times 10^{7} L_{\odot}$ by Dwek et al. (1995) and NIR to hard X-ray flux ratio, we can estimate the $17-60 \mathrm{keV}$ luminosity of the Galactic bulge as $(1.23 \pm 0.05) \times 10^{37} \mathrm{erg} \mathrm{s}^{-1}$. Assuming a Galactic bulge mass of $M_{\text {bulge }}=1-1.3 \times 10^{10} M_{\odot}$ (e.g., Dwek et al. 1995), we can estimate the unit stellar mass hard X-ray emissivity of the

${ }^{1}$ EGRET gamma-ray background map, $\mathrm{HI}$ and $\mathrm{CO}$ data were obtained from Skyview (http://skyview.gsfc.nasa.gov/) facility supplied by NASA/GSFC.

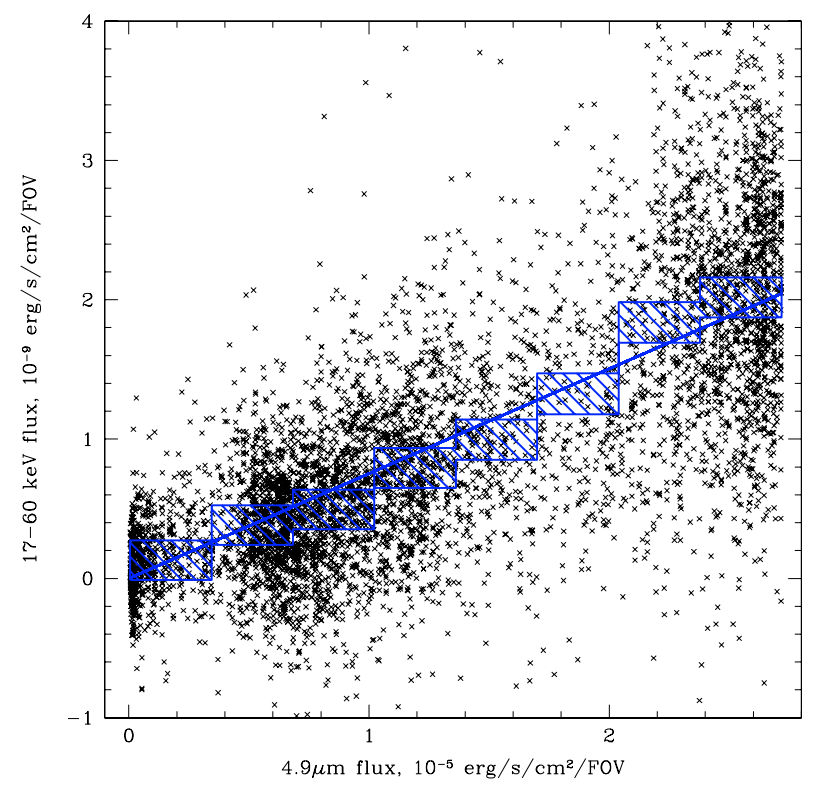

Fig. 10. Correlation of the near infrared measurements of $\mathrm{COBE} / \mathrm{DIRBE}$ at $4.9 \mu \mathrm{m}$ with the hard X-ray fluxes observed by IBIS/ISGRI in the energy band $17-60 \mathrm{keV}$. Every point corresponds to a single INTEGRAL/IBIS pointing. Value of axis $y$ is the GRXE flux measured by IBIS/ISGRI when pointed at a certain sky position, value of axis $x$ is the $4.9 \mu \mathrm{m}$ flux obtained by convolution of the DIRBE NIR map with the IBIS/ISGRI collimator response function. Scatter of points in " $y$ " direction is compatible with statistical and systematic uncertainties of IBIS/ISGRI measurements. Values obtained by averaging of hard X-ray measurements in NIR flux bins are shown by blue squares. The half height of the squares represents the uncertainty of our measurements. In most cases the uncertainty is dominated by systematic errors of our background model $\sim 10 \mathrm{mCrab}$. The solid blue line is the linear correlation of NIR and hard X-ray fluxes: $F_{17-60 \mathrm{keV}} / F_{4.9 \mu \mathrm{m}}=(7.52 \pm 0.33) \times 10^{-5}$.

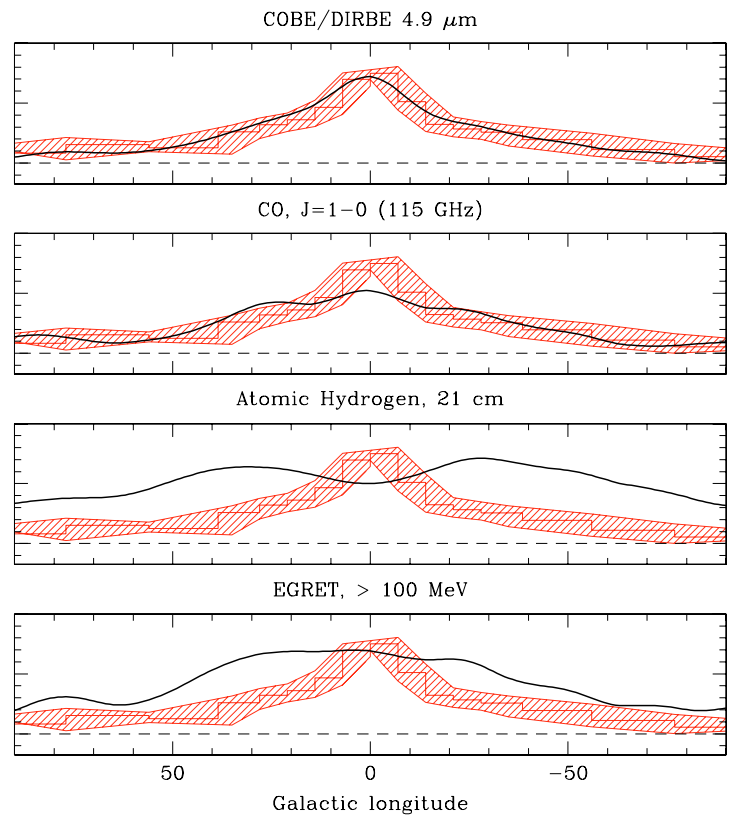

Fig. 11. Profile of the GRXE in hard X-rays (17-60 keV) observed by IBIS/ISGRI along with the profiles of EGRET gamma-ray background, neutral hydrogen (HI) emission, and molecular gas (CO) emission. All the profiles were convolved with the IBIS/ISGRI collimator response function and arbitrary normalized for better visibility. 


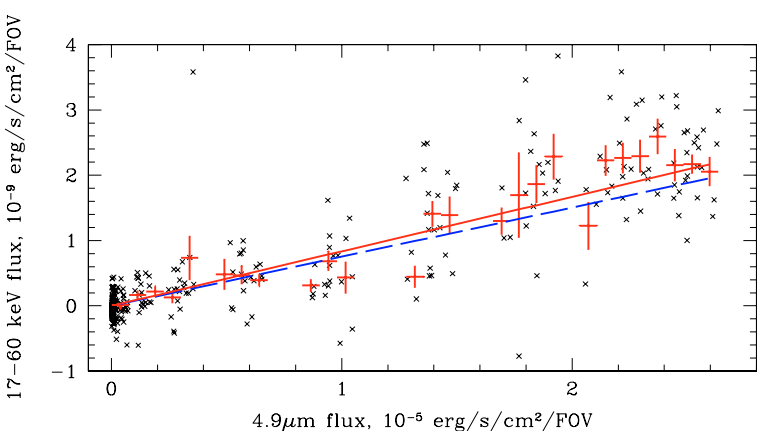

Fig. 12. Correlation between NIR (COBE/DIRBE, $4.9 \mu \mathrm{m})$ and hard X-ray (IBIS/ISGRI, 17-60 keV) fluxes. ISGRI detector count rate was measured using Galactic center latitude scans in "rocking mode" approach (background model "2", see text). Each black point represents individual measurement. ISGRI detector count rate was averaged over NIR flux as shown by red data points. Linear correlation coefficient was found as $(8.44 \pm 0.28) \times 10^{-5}$ (red line). Blue dashed line represents NIR to hard X-ray correlation obtained using all available observations (background model "1").

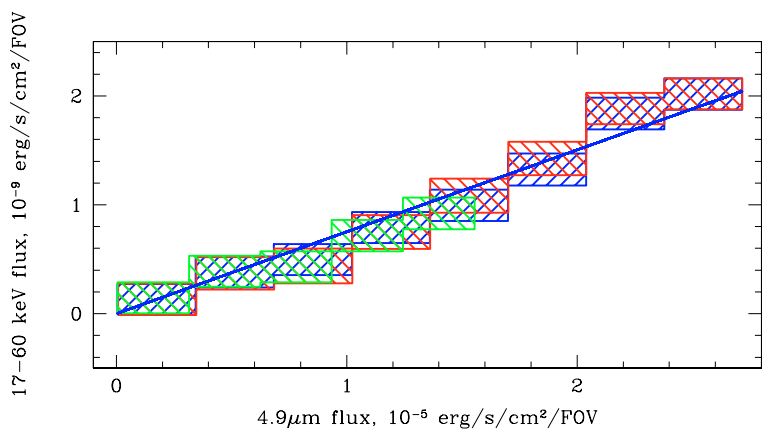

Fig. 13. NIR- and hard X-ray correlation (similar to Fig. 10) measured in the Galactic bulge $\left(|l|<10^{\circ}\right.$, red boxes) and Galactic disk $\left(|l|>20^{\circ}\right.$, green boxes) regions separately. The linear correlation between NIR and hard X-ray fluxes measured using all GRXE observations is shown in blue (shaded region and fit).

GRXE as $L_{17-60 \mathrm{kev}} / M_{\text {bulge }}=0.9-1.2 \times 10^{27} \mathrm{erg} / \mathrm{s} / M_{\odot}$. Taking the disk-to-bulge mass ratio to be $\sim 2$, we can estimate that the total hard X-ray (17-60 keV) luminosity of the Galaxy in the ridge emission is $(3.7 \pm 0.2) \times 10^{37} \mathrm{erg} \mathrm{s}^{-1}$.

\subsection{Spectrum}

Using the data from Galactic latitude scans, which have the smallest systematic uncertainties of the ISGRI background subtraction, we obtained the spectrum of the GRXE in the energy band $17-200 \mathrm{keV}$. It is presented in Fig. 14 together with the summed spectrum of all detected point sources in the Galactic center region. Note that after subtraction of bright point sources detected by IBIS/ISGRI, we do not detect any additional hard $\mathrm{X}$-ray emission at energies $\sim 60-200 \mathrm{keV}$. Our $2 \sigma$ upper limit on such emission is $\sim 60 \mathrm{mCrab}$ for the IBIS field of view in the 57-86 keV energy band. To demonstrate that the GRXE above $60 \mathrm{keV}$ vanishes, we present the latitude profile of the IBIS/ISGRI detector count rate in the energy band $57-86 \mathrm{keV}$ (Fig. 15). It should be noted that the observed cutoff in the GRXE spectrum above $60 \mathrm{keV}$ does not agree with the unresolved hard X-ray excess reported by Strong et al. (2005) and Bouchet et al. (2005) in the SPI data. The reason for this discrepancy is not yet understood. One can speculate that it might be related to significantly more coarse spatial resolution of SPI

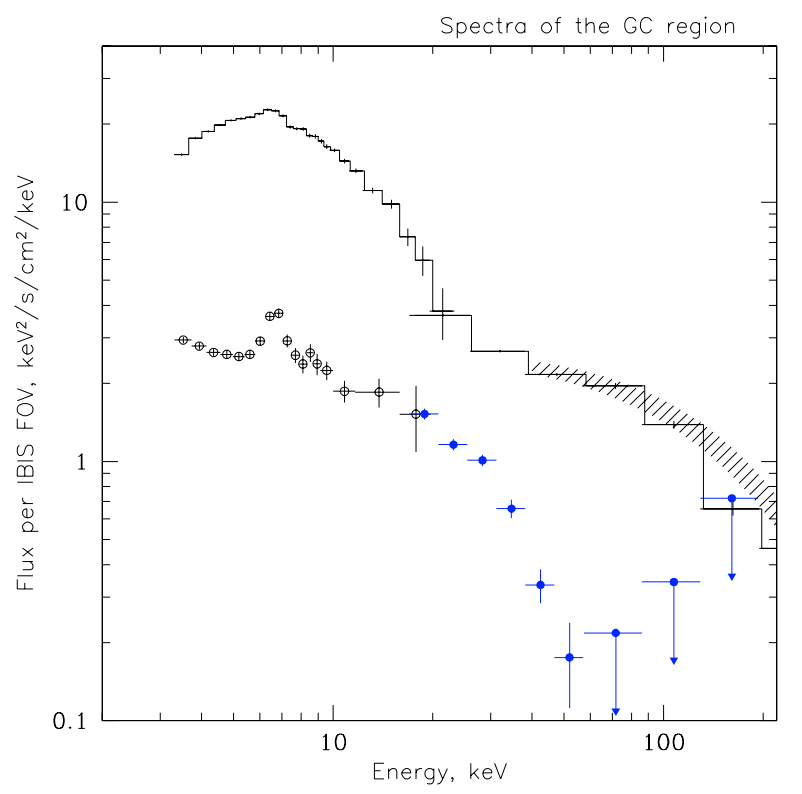

Fig. 14. Spectrum of the Galactic center region in the energy band 3-200 $\mathrm{keV}$ as it would be seen by a $\sim 15^{\circ} \times 15^{\circ}$ field-of-view instrument. The data points at energies $>17 \mathrm{keV}$ are obtained by IBIS/ISGRI. The arrows at energies $>60 \mathrm{keV}$ are $2 \sigma$-upper limits. The points at 3-20 keV were obtained from the data of RXTE/PCA (Galactic bulge scan data taken on March 15, 1999) and scaled to match the IBIS/ISGRI points at $\sim 20 \mathrm{keV}$. Circles show the spectrum of the GRXE, open circles - RXTE/PCA data, and filled circles - INTEGRAL/IBIS/ISGRI data. The histogram shows the integrated emission of detected point sources. The shaded region represents a model fit to the spectrum of the GC region measured by OSSE. The contribution of positron annihilation radiation, which consists of a $511 \mathrm{keV}$ line and annihilation continuum, was subtracted (Kinzer et al. 1999). Its normalization was scaled to match that of the IBIS/ISGRI points at $30-40 \mathrm{keV}$.

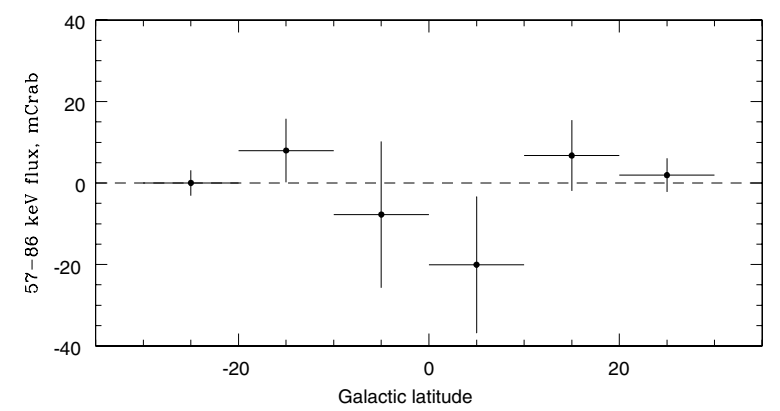

Fig. 15. Latitude profile of the GRXE in the energy band 57-86 keV. Profile is averaged over GRXE measurements in $|l|<5^{\circ}$. Only an upper limit on the ridge emission in this energy band can be obtained.

( 2.5 degrees). The correction for the contribution of bright point sources in the crowded fields (like GC) might be less accurate for SPI than for IBIS/ISGRI and some of the excess could be due the residual signal from point sources. A work on the detailed comparison of the SPI and IBIS data is now in progress and will be reported elsewhere.

After we showed that hard GRXE volume emissivity traces the stellar mass density in the Galaxy, we can construct a broadband unit stellar mass spectrum of the GRXE. We obtained ratios of hard X-ray surface brightness in each energy band to NIR surface brightness. NIR surface brightness was obtained after convolution of reddening-corrected COBE/DIRBE $4.9 \mu \mathrm{m}$ measurements with the IBIS collimator response function in appropriate energy bins. Using the obtained ratios and unit stellar mass 


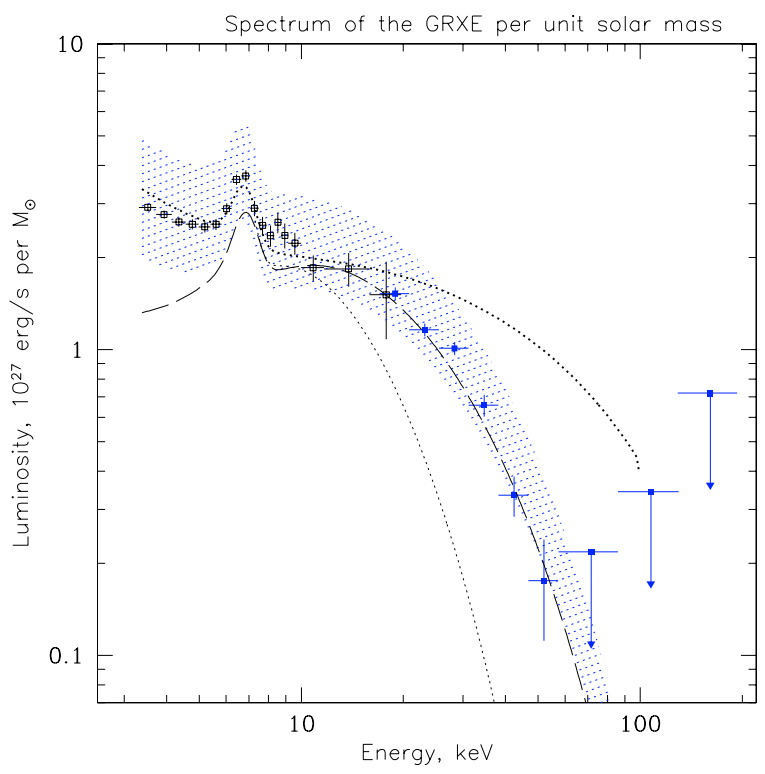

Fig. 16. Broad-band spectrum of GRXE per unit stellar mass. Blue points represent result of this work. Shaded region represents a "toy" composite spectrum of weak Galactic X-ray sources with weights according to Sazonov et al. (2006). For the input template spectrum of intermediate polars we adopted a white dwarf mass $M_{\mathrm{wd}}=0.5 M_{\odot}$. The approximate (due to uncertainties in the relative weights of CVs and coronally active stars) contribution of magnetic CVs (intermediate polars and polars) to the GRXE emissivity is shown by the dashed curve. For comparison the thin and thick dotted lines show the composite GRXE spectra calculated assuming the white draft masses of 0.3 and $1 M_{\odot}$ respectively.

$4.9 \mu \mathrm{m}$ luminosity we calculated the unit stellar mass hard X-ray spectrum of the GRXE (Fig. 16).

\section{Discussion}

\subsection{GRXE spectrum}

The obtained spectrum of the GRXE can now be compared with a composite spectrum of known types of weak Galactic X-ray sources. Unfortunately we do not have broad-band spectra of all sources that were used in the construction of the luminosity function of weak Galactic X-ray sources (Sazonov et al. 2006). Therefore we tried to obtain some "toy" composite spectra that would possess the main properties of the ideal sample of sources. As the input templates of spectra of individual classes of sources we take: the spectrum of V711 Tau as an RS CVn binary, AM Her as a polar, and SU UMa as a dwarf nova. For the spectrum of intermediate polars, which are the dominant contributors in hard X-rays, we adopt the model spectrum of Suleimanov et al. (2005) with the white dwarf mass $M_{\mathrm{wd}}=0.5 M_{\odot}$.

The important difference of this "toy" composite spectrum from that used in the work of Revnivtsev et al. (2005) is the value of the white dwarf mass in the intermediate polar binary system. The temperature of the optically thin plasma emitting X-ray radiation in the case of accreting magnetic CVs (in particular in intermediate polars, which dominate in hard X-rays) strongly depends on the mass of the white dwarf (e.g., Aizu 1973). In the range of masses $\sim 0.3-1.0 M_{\odot}$, the optically thin plasma temperature, which is a measure of the virial temperature of protons near the white dwarf, is approximately $k T \propto M^{1.6-1.7}$ (see, e.g., WD mass-radius relation in Nauenberg 1972). Therefore, it would be more reasonable to use the average mass of the white dwarfs in the Galaxy rather than some peculiar mass value. Revnivtsev et al. (2005) used the spectrum of the binary system V1223 Sgr, which harbors a white dwarf with mass $M_{\mathrm{wd}} \sim 1 M_{\odot}$ (e.g., Suleimanov et al. 2005), while the average mass of white dwarfs in the Galaxy is apparently considerably smaller $-M_{\mathrm{wd}} \sim 0.5 M_{\odot}$ (e.g., Bergeron et al. 1992, 1995; de Kool 1992; Politano 1996). Therefore, the hard X-ray part of the true spectrum of the GRXE if it were composed of $\sim 0.5 M_{\odot}$ white dwarfs is expected to be significantly softer than shown in Fig. 8 of Revnivtsev et al. (2005). This is indeed seen in Fig. 16. For illustration the spectrum generated for the lower end of the possible WD mass range $\left(M_{\mathrm{wd}}=0.3 M_{\odot}\right)$ is also shown in Fig. 16

Note that according to the above reasoning the shape of the GRXE spectrum in hard X-rays can be used to determine the average mass of the white dwarfs in accreting magnetic CVs in the Galaxy. The exact determination of the average WD mass is subject to uncertainties of relative contribution of different types of Galactic X-ray sources, but our first estimate shows that it is approximately consistent with $\left\langle M_{\mathrm{wd}, \mathrm{IP}}\right\rangle \sim 0.5 M_{\odot}$. At the energies 60-200 keV we did not detect any hard X-ray emission of the Galaxy apart from the contribution of a relatively small number of bright point sources visible by IBIS/ISGRI.

At energies higher than 100-200 keV a more detailed study of the IBIS/ISGRI detector background is needed to recover the properties of the unresolved emission in the Galaxy. In addition to the instrumental problems at these energies there is a strong contribution of the diffuse positronium continuum in the Galactic Center region, which should be carefully taken into account. We plan to study the unresolved Galactic continuum at these high energies in our future work.

\subsection{IGR J17456-2901}

Images of the Galactic center obtained by IBIS/ISGRI (e.g., Revnivtsev et al. 2004b; Bélanger et al. 2006) showed a bright spot at the position of the Galactic center $\left(\mathrm{Sgr} \mathrm{A}{ }^{*}\right.$ ), which was designated as an INTEGRAL source IGR J17456-2901. Association of this source with a low-mass X-ray binary located near Sgr A* (Revnivtsev et al. 2004b) or with emission from Sgr A* itself (e.g., Bélanger et al. 2004) does not allows one to explain the properties of the source. In particular, it was shown that this source is most likely not a point-like source and does not consist of a small number of bright LMXBs (Neronov et al. 2005). Bélanger et al. (2006) argued that the emission of IGR J17456-2901 might have the same origin as the source of ultra-high energy (TeV) photons seen by HESS (Aharonian et al. 2006).

It was argued by Revnivtsev et al. (2005) based on the proportionality of the GRXE volume emissivity to the Galactic stellar mass density that the emission of IGR J17456-2901 should consist (or contain a large contribution) of the integrated (due to the limited angular resolution of IBIS/ISGRI) emission of all the stars $\left(\sim 10^{8} M_{\odot}\right)$ within central $\sim 30$ pc $\left(\sim 12^{\prime}\right.$ at the Galactic center distance, which is approximately equal to the INTEGRAL/IBIS angular resolution) around Sgr A*. Therefore, we could anticipate that the spectrum of IGR J17456-2901 would be similar to that of the true GRXE (e.g., Revnivtsev et al. 2005). This is partially true (see Fig. 17).

The spectrum of the central $\sim 10^{\prime}$ around $\mathrm{Sgr} \mathrm{A}^{*}$ is very similar to that of the GRXE at least in the energy band $\sim 3-30 \mathrm{keV}$, except for the somewhat higher normalization, which would correspond better to an integrated stellar mass $\sim 2 \times 10^{8} M_{\odot}$ rather then $10^{8} M_{\odot}$ as we assumed. Whether this is just due to the 


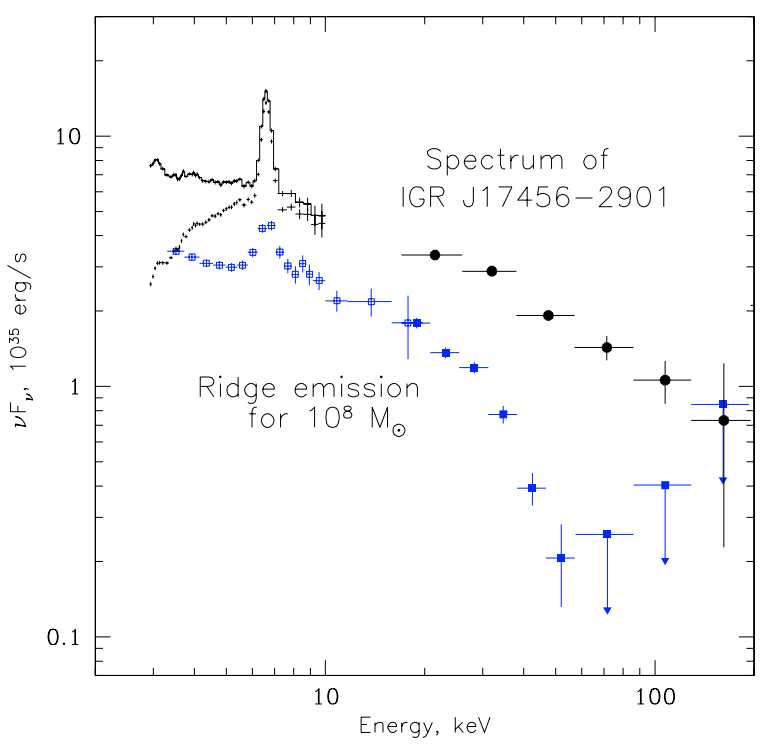

Fig. 17. The spectrum of the Galactic ridge X-ray emission calculated for a $10^{8} M_{\odot}$ stellar mass (blue squares). The spectrum of Galactic center source IGR J17456-2901 is shown by black symbols. Measurements by INTEGRAL/IBIS are shown by filled symbols. The hard X-ray spectrum is complemented by a spectrum of integrated emission of the central $10^{\prime}$ around Sgr $\mathrm{A}^{*}$, measured by ASCA/GIS (lower spectrum showed by black crosses) and corrected for interstellar absorption $N_{\mathrm{H}}=7 \times 10^{22} \mathrm{~cm}^{-2}$ (upper spectrum showed by black crosses).

uncertain value of the total stellar mass within $\sim 30 \mathrm{pc}$ around Sgr A* (e.g., Lindqvist et al. 1992), or in fact due to a different value of X-ray emissivity per unit stellar mass in this area ${ }^{2}$ is the subject of a special detailed study that is beyond the scope of this paper. However, as can be seen from Fig. 17, the spectrum of IGR J17456-2901 at energies $60-100 \mathrm{keV}$ is obviously harder than that of the GRXE. Below we outline several possible origins of the observed spectral difference.

\section{Confusion?}

The surroundings of the Galactic center is a very complex region populated by different types of compact and diffuse sources of emission. The telescope IBIS, whose data were used here to derive the spectrum of IGR J17456-2901, has angular resolution $\sim 12^{\prime}$, therefore we cannot exclude that some emission region spatially distinct from the nuclear stellar cluster contributes to the flux that we measure from the position of IGR J17456-2901.

This possibility finds relatively solid support from the fact that the peak of hard X-ray emission at energies $>70-80 \mathrm{keV}$, where the contribution of compact sources visible at lower energies vanishes, has a large offset with respect to $\mathrm{Sgr} \mathrm{A*}-\sim 5.7^{\prime}$ (see also Bélanger et al. 2006). At the peak of the hard X-ray emission there are no known persistently bright compact X-ray sources (see, e.g., Sakano et al. 2002), while high energy (GeV and $\mathrm{TeV}$ ) non-thermal emission has been observed, presumably originating as a result of interaction of cosmic rays with interstellar matter (Aharonian et al. 2006). If the spectrum of this non-thermal emission has a relatively flat slope (e.g., $\Gamma \sim 2$ ), then its relative contribution in the standard X-ray energy band $(<10 \mathrm{keV})$ should be very small and should strongly rise with energy, therefore leading to the observed difference in the spectra

2 We would like to note that for the near-infrared spectral band, the mass-to-light ratio was found to be different within central $30 \mathrm{pc}$ around Sgr A* and in the rest of the Galaxy, see, e.g., Launhardt et al. (2002). of IGR J17456-2901 and the GRXE. Another example of possible confusion of different sources is a strongly photoabsorbed hard X-ray source, which is bright in hard X-rays but practically absent in the standard X-ray band, located at $6^{\prime}$ of Sgr A*. If, to the contrary, the hard X-ray emission of IGR J17456-2901 does belong to the Galactic nuclear stellar cluster, then different possible scenarios may be proposed.

Additional population of sources in the nuclear stellar cluster?

The nuclear stellar cluster is one of the densest regions in the Galaxy. The stellar density within a few parsecs of Sgr A* exceeds $10^{5}$ stars $\mathrm{pc}^{-3}$. It is not unreasonable to assume that such an exotic environment may provide conditions for formation of a population of X-ray emitting systems different from those in the rest of the Galaxy. Tens or hundreds of sources per the $\sim 10^{8} M_{\odot}$ stellar mass of the central $30 \mathrm{pc}$ with luminosities $\sim 10^{32} \mathrm{erg} / \mathrm{s}$ and hard X-ray spectra could significantly contribute to the observed difference between the spectra of the GRXE and IGR J17456-2901.

\section{Massive white dwarfs?}

If the average mass of accreting white dwarfs in the Galactic center region is systematically higher than in the rest of the Galaxy, we can also anticipate a considerably harder cumulative spectrum of weak X-ray sources. Induced massive white dwarf binaries formation due to tidal capture process in dense environment, similar to that in globular clusters (e.g., Ivanova et al. 2006), is not very likely due to the much higher velocities of stars in the nuclear stellar cluster. In principle, more massive white dwarfs might concentrate in regions with deeper gravitational potential due to gravitational mass segregation, similar to what is observed in globular clusters (e.g., Grindlay 2006), but in the case of our Galactic center this scenario is also not very likely (see, e.g., simulations in Freitag et al. 2006).

\section{Conclusion}

1) We have shown that the surface brightness distribution of the GRXE in the energy band 17-60 keV very closely follows the near infrared surface brightness distribution throughout the Galaxy. This strongly supports the conclusion of Revnivtsev et al. (2005) based on lower energies (3-20 keV) data. The surface brightness distributions of the gamma-ray background (EGRET data, $30 \mathrm{MeV}-10 \mathrm{GeV}$ ), neutral interstellar matter (HI map), and molecular interstellar gas (CO map) do not show such correspondence with the hard GRXE intensity. The hard X-ray (17-60 keV) emissivity of the Galactic ridge, recalculated per unit stellar mass is $(0.9-1.2) \times 10^{27} \mathrm{erg} \mathrm{s}^{-1} M_{\odot}^{-1}$. This value is in good agreement (after correction for the energy band) with the unit stellar mass X-ray emissivity of weak Galactic X-ray sources (Sazonov et al. 2006; Revnivtsev et al. 2005). The total Galactic hard X-ray luminosity of the GRXE is $(3.7 \pm 0.2) \times$ $10^{37} \mathrm{erg} \mathrm{s}^{-1}$ in the $17-60 \mathrm{keV}$ energy band.

However, we should note that the difference in the morphology of the EGRET Galactic gamma-ray background and hard X-ray (17-60 keV) ridge emission observed by IBIS/ISGRI cannot by itself be considered as a strong argument against the hypothesis of the cosmic ray origin for the GRXE. Indeed, if the hard X-ray background emission of the Galaxy were dominated by bremsstrahlung of low energy $(\leqslant 0.5 \mathrm{MeV})$ cosmic ray electrons (see, e.g., Stecker 1977; Mandrou et al. 1980; Sacher \& Schoenfelder 1984; Harris et al. 1990), then these electrons might be confined to an almost immediate vicinity of their birthplace if the interstellar magnetic field is sufficiently tangled. 


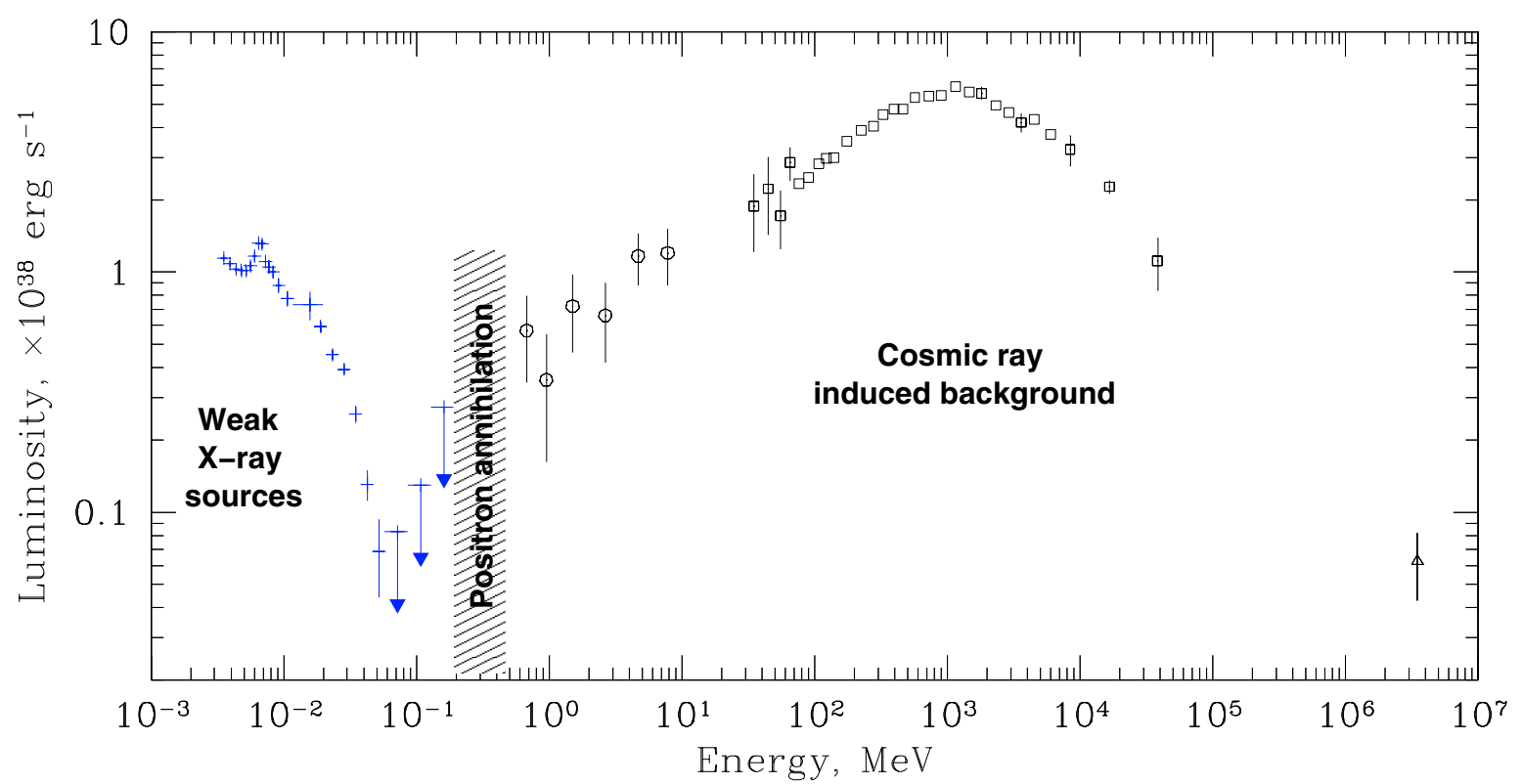

Fig. 18. Schematic luminosity spectrum of "unresolved" emission of the Galaxy in the energy band 3 keV-4 TeV. In the X-ray energy band the luminosity spectrum was scaled from the GRXE unit stellar mass emissivity spectrum (Fig. 16) assuming a mass of the Galaxy of $3.9 \times 10^{10} M_{\odot}$. For scaling the $\gamma$-ray part of the spectrum we adopted a value of the total Galactic luminosity at $>100 \mathrm{MeV}$ of $L>100 \mathrm{MeV}=2 \times 10^{39} \mathrm{erg} / \mathrm{s}(\mathrm{Bloemen}$ et al. 1984). Measurements in $\gamma$-rays by CGRO/OSSE and CGRO/EGRET are adopted from Kinzer et al. (1999), the measurement at TeV energies is rescaled from Atkins et al. (2005); Prodanovic et al. (2006). The shaded region at energies $\sim 200-500 \mathrm{keV}$ denotes the area where positron annihilation radiation in the Galactic center region strongly dominates.

This would happen because electrons at these energies have very small mean free paths in the presence of tangled interstellar magnetic field (e.g., Zwickl \& Webber 1978). If the places of origin of such electrons somehow followed the stellar mass distribution in the Galaxy, then the hard X-ray background, induced by such cosmic-rays would also follow the NIR intensity distribution.

2) Subtracting the flux of detected point sources from the total IBIS aperture sky flux we have obtained the spectrum of the GRXE. Its shape well agrees with the spectral shape of accreting magnetic white dwarfs, which are expected to provide a dominant contribution to the Galactic X-ray emission in this energy band. The shape of the spectrum of the GRXE allows us to estimate the average mass of accreting magnetic white dwarfs in the Galaxy $\left\langle M_{\mathrm{wd}}\right\rangle \sim 0.5 M_{\odot}$.

3) We have shown that the Galactic background emission is undetectable in the energy range $\sim 60-200 \mathrm{keV}$. The signal that was previously ascribed to the Galactic background emission at these energies was most likely due to emission of unresolved point sources.

4) Our results fit in the model in which the Galactic ridge $\mathrm{X}$-ray emission in energy band $3-100 \mathrm{keV}$ originates as the superposition of weak Galactic point sources. This suggests that at energies $\gtrsim 200 \mathrm{keV}$ a change of the nature of the unresolved Galactic emission to cosmic-ray induced background should occur. To illustrate this we present a scheme of the luminosity spectrum of unresolved emission of the whole Galaxy in Fig. 18. One should remember that according to our model the ratio of the $\gamma$ ray to $\mathrm{X}$-ray unresolved background luminosities strongly varies across the Galaxy; therefore the presented broad-band spectrum should be considered as only a schematic representation of the real luminosity spectrum of the whole Galaxy.

As the Galactic center region at energies $200-500 \mathrm{keV}$ contains a powerful diffuse emission of the positronium continuum that is very hard to disentangle from the cosmic-ray induced radiation, we anticipate that an answer to the question where the cosmic-ray induced radiation begins to dominate can be obtained only either by studying regions away from the Galactic center or at energies $0.5-10 \mathrm{MeV}$.

Acknowledgements. This research was done thanks to unique capabilities of the INTEGRAL observatory. The data used were obtained from the European and Russian INTEGRAL Science Data Centers and from the High Energy Astrophysics Science Archive Research Center Online Service of the NASA/Goddard Space Flight Center. We acknowledge the use of the Legacy Archive for Microwave Background Data Analysis (LAMBDA). Support for LAMBDA is provided by the NASA Office of Space Science. Authors thank Hans Ritter for useful discussion regarding WD masses in the Galaxy. The work was supported by the President of the Russian Federation (through the program of support of leading scientific schools, project NSH-1100.2006.2), by the Presidium of the Russian Academy of Sciences/RAS (the program "Origin and evolution of stars and galaxies"), by the Division of Physical Sciences of the RAS (the program "Extended objects in the Universe"), and by the Russian Basic Research Foundation (the project 05-02-16540).

\section{References}

Aharonian, F., Akhperjanian, A. G., Bazer-Bachi, A. R., et al. 2006, Nature, 439, 695

Aizu, K. 1973, PThPh, 49, 1184

Atkins, R., Benbow, W., Berley, D., et al. 2005, PhRvL, 95, 251103

Bélanger, G., Goldwurm, A., Goldoni, P., et al. 2004, ApJ, 601, L163

Bélanger, G., Goldwurm, A., Renaud, M., et al. 2006, ApJ, 636, 275

Bergeron, P., Saffer, R. A., \& Liebert, J. 1992, ApJ, 394, 228

Bergeron, P., Liebert, J., \& Fulbright, M. S. 1995, ApJ, 444, 810

Bouchet, L., Roques, J. P., Mandrou, P., et al. 2005, ApJ, 635, 1103

Bloemen, J. B. G. M., Blitz, L., \& Hermsen, W. 1984, ApJ, 279, 136

Bloser, P. F., Narita, T., Jenkins, J. A., et al. 2002, SPIE, 4497, 88

Churazov, E., Sunyaev, R., Sazonov, S., Revnivtsev, M., \& Varshalovich, D. 2005, MNRAS, 357, 1377

Churazov, E., Sunyaev, R., Revnivtsev, M., et al. 2006

[arXiv: astro-ph/0608250]

Dwek, E., Arendt, R. G., Hauser, M. G., et al. 1995, ApJ, 445, 716

Ebisawa, K., Tsujimoto, M., Paizis, A., et al. 2005, ApJ, 635, 214

Freitag, M., Amaro-Seoane, P., \& Kalogera, V. 2006

[arXiv: astro-ph/0603280]

Gehrels, N., Barthelmy, S. D., Teegarden, B. J., et al. 1991, ApJ, 375, L13 
Grimm, H.-J., Gilfanov, M. \& Sunyaev, R. 2002, A\&A, 391, 923

Grindlay, J. E. 2006 [arXiv: astro-ph/0605133]

Hajdas, W., Bühler, P., Eggel, C., et al. 2003, A\&A, 411, L43

Hands, A. D. P., Warwick, R. S., Watson, M. G., \& Helfand, D. J. 2004, MNRAS, 351,31

Harris, M. J., Share, G. H., Leising, M. D., Kinzer, R. L., \& Messina, D. C. 1990, ApJ, 362, 135

Hunter, S. D., Bertsch, D. L., Catelli, J. R., et al. 1997, ApJ, 481, 205

Indebetouw, R., Mathis, J. S., Babler, B. L., et al. 2005, ApJ, 619, 931

Ivanova, N., Heinke, C. O., Rasio, F. A., et al. 2006, MNRAS, 372, 1043

Kinzer, R. L., Purcell, W. R., \& Kurfess, J. D. 1999, ApJ, 515, 215

Kniffen, D. A., Bertsch, D. L., Morris, D. J., Palmeira, R. A. R., \& Rao, K. R. 1978, ApJ, 225, 591

Knödlseder, J., Jean, P., Lonjou, V., et al. 2005, A\&A, 441, 513

de Kool, M. 1992, A\&A, 261, 188

Kraushaar, W. L., Clark, G. W., Garmire, G. P., et al. 1972, ApJ, 177, 341

Launhardt, R., Zylka, R., \& Mezger, P. G. 2002, A\&A, 384, 112

Lebrun, F., Terrier, R., Bazzano, A., et al. 2004, Nature, 428, 293

Leventhal, M., MacCallum, C. J., \& Stang, P. D. 1978, ApJ, 225, L11

Lindqvist, M., Habing, H. J., \& Winnberg, A. 1992, A\&A, 259, 118

Lutz, D., Genzel, R., Sternberg, A., et al. 1996, A\&A, 315, L137

Mandrou, P., Bui-van, A., Vedrenne, G., \& Niel, M. 1980, ApJ, 237, 424

Nauenberg, M. 1972, ApJ, 175, 417

Neronov, A., Chernyakova, M., Courvoisier, T. J., \& Walter, R. 2005 [arXiv: astro-ph/0506437]

Politano, M. 1996, ApJ, 465, 338

Prodanovic, T., Fields, B. D., \& Beacom, J. F. 2006, Astropart. Phys., 27, 10

Purcell, W. R., Bouchet, L., Johnson, W. N., et al. 1996, A\&AS, 120, 389
Reglero, V., Sánchez, F., Rodrigo, J., et al. 2001, Exploring the gamma-ray universe, Proceedings of the 4th INTEGRAL Workshop, 4-8 September 2000, Alicante, Spain, ed. B. Battrick, 619

Revnivtsev, M. G., Sunyaev, R. A., Varshalovich, D. A., et al. 2004, AstL, 30, 382

Revnivtsev, M., Sazonov, S., Gilfanov, M., Churazov, E., \& Sunyaev, R. 2005 [arXiv:astro-ph/0510050]

Sacher, W., \& Schoenfelder, V. 1984, ApJ, 279, 817

Sakano, M., Koyama, K., Murakami, H., Maeda, Y., \& Yamauchi, S. 2002, ApJS, 138,19

Sazonov, S., Revnivtsev, M., Gilfanov, M., Churazov, E., \& Sunyaev, R. 2006, A\&A, 450, 117

Skibo, J. G., \& Ramaty, R. 1993, A\&AS, 97, 145

Skibo, J. G., Johnson, W. N., Kurfess, J. D., et al. 1997, ApJ, 483, L95

Stecker, F. W. 1973, ApJ, 185, 499

Stecker F. W., 1977, ApJ, 212, 60

Strong, A. W., Diehl, R., Halloin, H., et al. 2005, A\&A, 444, 495

Sugizaki, M., Mitsuda, K., Kaneda, H., et al. 2001, ApJS, 134, 77

Suleimanov, V., Revnivtsev, M., \& Ritter, H. 2005, A\&A, 435, 191

Terrier, R., Lebrun, F., Bazzano, A., et al. 2003, A\&A, 411, L167

Terrier, R., Lebrun, F., Belanger, G., et al. 2004, Proceedings of the 5th INTEGRAL Workshop, Munich 16-20 February 2004, ESA SP-552, 513 Ubertini, P., Lebrun, F., Di Cocco, G., et al. 2003, A\&A, 411, L131

Vedrenne, G., Roques, J.-P., Schönfelder, V., et al. 2003, A\&A, 411, L63

Worrall, D. M., Marshall, F. E., Boldt, E. A., \& Swank, J. H. 1982, ApJ, 255, 111

Winkler, C., Courvoisier, T. J.-L., Di Cocco, G., et al. 2003, A\&A, 411, L1

Zwickl, R. D., \& Webber, W. R. 1978, JGR, 83, 1157 